\title{
Evaluation of Online Advertisement Design Using Near-infrared Spectroscopy
}

\author{
Chikaho Kurahashi, ${ }^{1}$ Tadanobu Misawa, ${ }^{2 *}$ and Kazuya Yamashita ${ }^{2}$ \\ ${ }^{1}$ Graduate School of Science and Engineering for Education, University of Toyama, \\ 3109 Gofuku, Toyama-shi, Toyama 930-8555, Japan \\ ${ }^{2}$ Graduate School of Science and Engineering, University of Toyama, \\ 3109 Gofuku, Toyama-shi, Toyama 930-8555, Japan
}

(Received December 26, 2017; accepted April 9, 2018)

Keywords: near-infrared spectroscopy, neuromarketing, online advertisement, placement, color

Sales from online advertisements have increased, and this market is expected to expand further with the spread of the Internet of Things (IoT). To enable more effective marketing, it is important to consider the advertisement's design. In general, questionnaires are typically used to evaluate the marketing effectiveness. However, neuromarketing based on brain activity has attracted attention in recent years as a new marketing method. In this study, we examined the effects of advertisement rating, placement, and color on brain activity using near-infrared spectroscopy (NIRS). For that, we used 34 pictorial advertisements, 34 verbal advertisements, and 34 webpages on which the two advertisement types were placed on the right and the left sides of webpage content. First, seven participants viewed the 34 webpages. Subsequently, they viewed the 68 advertisements and rated them on a 7-point scale. Brain activity was measured simultaneously while they viewed the advertisements. The NIRS results indicated that the concentration of oxygenated hemoglobin increased when the participants viewed highly rated advertisements compared with poorly rated ones. In addition, a significant difference in brain activity was also observed in accordance with the placement and color of the pictorial advertisements and the color of verbal advertisements.

\section{Introduction}

In recent years, online advertisement has attracted attention owing to the spread of the internet and smartphones. The reasons behind this spread are advantages such as low-cost usage fees, easy and quick information sharing, specific target search based on keywords, and possible overview of effectiveness based on the number of clicks or viewings. Furthermore, being able to spread the word broadly (to the whole world) is another advantage. Sales of online-advertised goods are increasing every year, and the market is expected to expand further with the spread of the Internet of Things (IoT), which is a network where various items are connected to the Internet. With the spread of IoT, it becomes easier to gather information such as consumer attributes and action history. The IoT has also enabled the displaying of

*Corresponding author: e-mail: misawa@eng.u-toyama.ac.jp http://dx.doi.org/10.18494/SAM.2018.1879 
advertisements not only on PCs and smartphones but also on various IoT devices. As a result, it has become possible to present more targeted advertisements such as displaying advertisements of sports drinks when passing in front of vending machines while jogging and displaying advertisements of diet goods on bathroom scales.

Many reports on online advertisements have appeared, including investigations of the differences in the ratings depending on the placement of pictorial and verbal advertisements, ${ }^{(1)}$ and the relationship between the advertisement's color and click-through rate. ${ }^{(2)}$ In addition, it has been reported that the advertisement's design influences consumers' purchasing intentions through advertising and brand attitudes. ${ }^{(3)}$ Therefore, for more effective marketing, it is important to consider the advertisement's design, such as the placement and color of the scheme. Although there are many studies on the relationship between color and advertisement, those in which the relationship is examined using brain information are few. In the previous advertising evaluation, it was common to use the click-through rate (CTR) and questionnaires as indicators. However, users of websites tend to ignore advertisements and CTR has been sluggish in recent years, so it is reported that CTR is not an effective measurement method. ${ }^{(4)}$ In addition, even if consumers do not remember advertisements, there are reports that improvements in purchase intentions and advertisement-product relationships have been observed, even three months after seeing the advertisement. ${ }^{(5)}$ Therefore, there is a possibility that sufficient advertisement evaluation cannot be performed with only the CTR. As for the questionnaire, the problem that the result and the consumer behavior are different is often pointed out. Consequently, the questionnaire may not be a sufficient advertisement evaluation method. Therefore, in this research, we focused on neuromarketing using brain activity as an evaluation method of advertisement.

Neuromarketing is the attempt to study the consumers' unconscious decision-making using brain activity. For example, in recent years, studies have been conducted using brain activity as an evaluation index, such as the evaluation of video advertisements using electroencephalography (EEG). ${ }^{(6)}$ Originally, from an economic perspective, decision-making and economic behaviors have been analyzed on the premise that consumers are rational. However, behavioral economics research has demonstrated that the consumers are not always rational. ${ }^{(7,8)}$ Thus, problems such as inconsistencies in the collected data and actual consumer behavior are often highlighted in traditional market surveys. Neuromarketing is considered to have the potential to solve these problems and improve the quality of marketing by estimating the consumers' unconscious decision-making pattern.

In view of these factors, it is important to evaluate and propose marketing designs based on brain activity to develop more effective online advertisements. Therefore, the purpose of this study was to examine the effect of an advertisement's rating, placement, and color on brain activity using pictorial and verbal advertisements as a foothold for the evaluation and proposal of better advertisement designs based on brain activity.

Brain activity measurements include EEG, functional magnetic resonance imaging, and near-infrared spectroscopy (NIRS). Among these, we chose NIRS because it is the most compact, easy to wear, and the least restricted by body movements and is thus suitable for measuring brain activity during normal tasks such as decision-making. 


\section{Materials and Methods}

\subsection{Brain activity measurements}

The NIRS measurements were conducted using the OEG-16 system (Spectratech Inc., Yokohama, Japan) with 16 channels and a $0.08 \mathrm{~s}$ sampling interval. The measurement area was limited to the prefrontal region (Fig. 1). The numbers in Fig. 1 represent each of the 16 channels.

While changes in the concentrations of both oxygenated hemoglobin (oxy- $\mathrm{Hb}$ ) and deoxygenated hemoglobin (deoxy-Hb) were measured using NIRS, we only used the changes in oxy-Hb concentration in the data analysis.

\subsection{Outline of the experiment}

Seven right-handed participants (three women and four men) aged 22 and 23 years participated in this study. All the participants were students of the Faculty of Engineering at the University of Toyama.

\subsection{Stimuli}

In total, 68 online advertisements (34 pictorial and 34 verbal) were used. Examples of the stimuli are shown in Fig. 2. A pictorial advertisement mainly consisted of a picture, while a verbal advertisement consisted mainly of words. These advertisements were especially selected on the basis of a pre-assessment session involving 13 volunteers (four women and nine men) aged 22 and 23 years. In the pre-assessment, volunteers rated 160 advertisements (80 pictorial and 80 verbal) on a 7-point scale. After that, we selected advertisements with small variations in rating among the volunteers. All stimuli were displayed on a 27-inch monitor.

\subsection{Experimental task}

The experimental task was designed on the basis of a previous study. ${ }^{(1)}$ Briefly, participants rated advertisements after viewing the webpages where two advertisements were placed on the

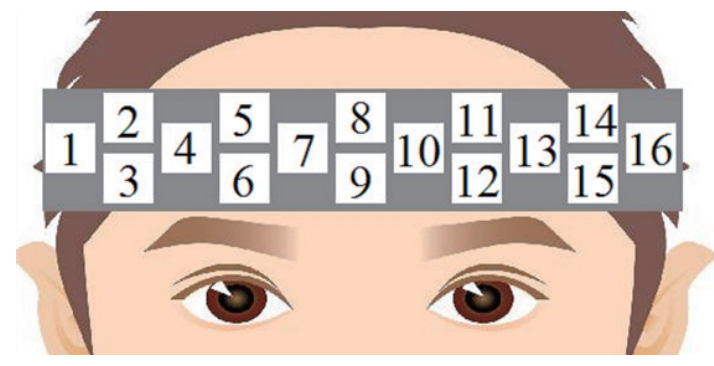

Fig. 1. (Color online) Measurement area.
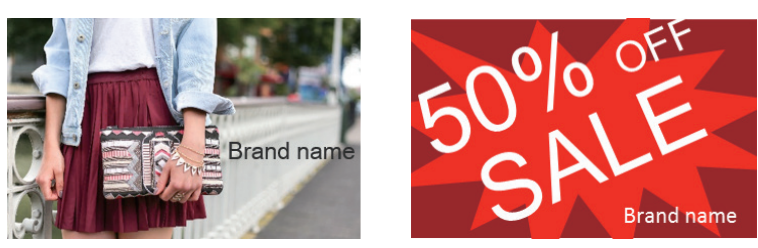

Fig. 2. (Color online) Examples of stimuli. These are not advertisements actually used in experiments but images created by the authors. 
right- and left-hand sides of webpage content (Fig. 3). The average value of an advertisement's rating in the pre-assessment was almost the same on both the right- and left-hand sides of the webpage for each case of the pictorial (average of right, 4.33; left, 4.38) and verbal (average of right, 3.26; left, 3.26) advertisements. The flow of the experimental task is given below.

Step 1. This step was a resting period of $5 \mathrm{~s}$ to stabilize brain activity. The letter " $\mathrm{X}$ " was displayed on the screen during this step.

Step 2. The webpage was displayed on the screen for $15 \mathrm{~s}$, followed subsequently by step 1 . The process proceeded to step 3 after the presentation of 34 webpages.

Step 3. This step was a resting period of $10 \mathrm{~s}$ to stabilize brain activity. The letter "X" was displayed on the screen during this step.

Step 4. An advertisement was displayed on the monitor for $10 \mathrm{~s}$.

Step 5. The participants rated the attractiveness of each advertisement on a 7-point scale. Subsequently, the participants returned to step 3. The task was terminated after the presentation of 64 advertisements.

Brain activity was measured during steps $3-5$. It has been previously reported that the concentration of oxy-Hb increases $3 \mathrm{~s}$ after stimulation, and the peak occurs after roughly $7.5 \mathrm{~s} .{ }^{(9)}$ Therefore, the advertisement evaluation based on brain activity (step 4) was set to $10 \mathrm{~s}$.

The main purpose of this task was to enable the participants to evaluate the advertisement placement unconsciously. Therefore, in steps 1 and 2, the participants were not informed that the experiment was related to advertisements. Instead, they were informed that the experiment was related to webpages, and were instructed to pay attention to the text in the middle of the webpage.

\subsection{Analysis}

We analyzed the NIRS data of channels 2-15 and excluded channels 1 and 16 because they included excessive artifacts. In addition, one participant was excluded from the analysis because he exhibited significantly different results compared with the other participants.

The data used in the analysis totaled approximately $10 \mathrm{~s}$ of advertisement viewing time and were collected during step 4 of the task with a sampling interval of $0.08 \mathrm{~s}$; there were 125 data points in total. We preprocessed the changes in the oxy-Hb concentration for each participant and each channel. The flow of the signal processing is shown below.

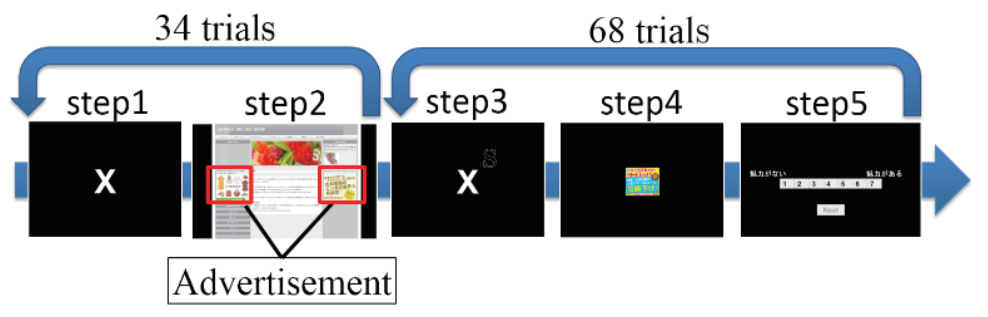

Fig. 3. (Color online) Experimental task. 
1. The changes in oxy-Hb concentration owing to body motion were removed by the hemodynamic modality separation (HMS) method provided by Spectratech Inc.

2. All data were converted to a frequency domain by fast Fourier transform (FFT). In addition, signals less than $0.01 \mathrm{~Hz}$ and greater than $0.7 \mathrm{~Hz}$ were cut using a band pass filter (BPF) to remove noise.

3. The z-score [average, 0 ; standard deviation (SD), 1] was calculated for the comparison among participants.

4. Data from step 4 were extracted and the direction of the vertical axis (oxy-Hb concentration) was shifted in such a way that the first data point of step 4 would be zero (the origin of the coordinate axes).

The HMS method is the latest technology associated with skin blood flow separation and was developed by the National Institute of Advanced Industrial Science and Technology. This method has been described in a recent study ${ }^{(10)}$ in which the function provided by Spectratech Inc. was used.

The heart rate $(0.7-1 \mathrm{~Hz})$ greatly affects the cerebral blood flow data measured using NIRS. ${ }^{(11)}$ In addition, in studies using NIRS, signals of less than $0.01 \mathrm{~Hz}$ are often cut to remove small changes (trends) ${ }^{(12)}$ thus, we used BPF $(0.01-0.7 \mathrm{~Hz})$ in this study.

\section{Results}

We examined the effects of the advertisement's rating, placement, and color on brain activity. First, data were divided into groups for each result and the normality test was conducted for each of 125 data points. Then $t$-test was conducted for data points with normality. Analysis was performed on oxy-Hb data for six people. Therefore, the results shown are not averages for each participant, but averages for six participants.

\subsection{Difference in brain activity depending on the advertisement's rating}

The changes in the oxy- $\mathrm{Hb}$ concentration of the six participants in step 4 were divided into low ratings and high ratings and were averaged for each channel. We defined ratings 1 and 2 as low and ratings 6 and 7 as high on the 7-point scale questionnaire. The sample sizes of the low and high ratings were 132 and 69, respectively.

Figure 4 depicts the results of this analysis. We observed significant differences between the low and high ratings groups using the $t$-test. The vertical bar represents the result of our $t$-test at a significance level of 5\%. A significant difference was observed in channels 5, 8, and 11 . In addition, similar trends were observed for channels 4, 6-10, and 13, although they were not statistically significant.

\subsection{Differences in brain activity depending on the advertisement's placement}

The changes in the oxy-Hb concentration of the six participants in step 4 were divided into pictorial and verbal advertisements. These changes were further subdivided into advertisements 


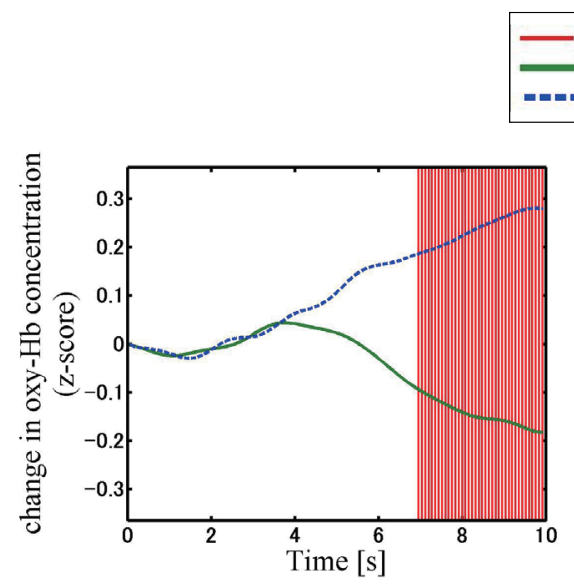

(a)

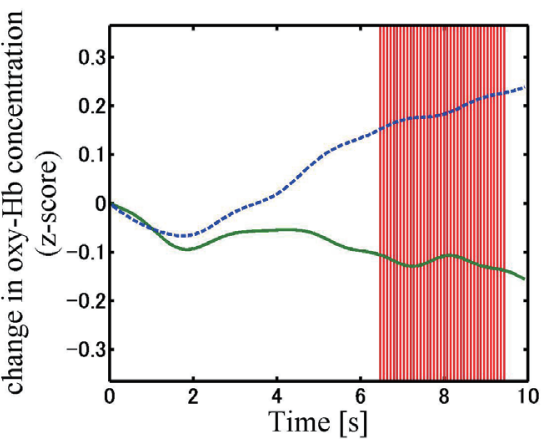

(b)

Fig. 4. (Color online) Example of difference in brain activity depending on evaluation. (a) ch 5 and (b) ch 8.

placed on the right and the left side of the webpage. Subsequently, the data from the six participants were averaged for each channel. The sample size of each group was 102. The average ratings for the pictorial and verbal advertisements were 2.99 (SD, 1.54) and 4.3 (SD, 1.54), respectively.

The $t$-test was conducted for left side versus right side for the pictorial and verbal advertisements. The results from the pictorial advertisements are shown in Fig. 5. Significant differences were observed in channel 2. In contrast, no significant differences were observed in the results of the verbal advertisements.

\subsection{Difference in brain activity depending on the advertisement's color}

To analyze the effect of color, the advertisements were classified into three classes using the average values of red (R), green $(\mathrm{G})$, and blue (B). The method used for classification was $k$-means clustering. The data were distributed as follows: class 1 included nine verbal advertisements and 11 pictorial advertisements, class 2 included 14 verbal advertisements and 11 pictorial advertisements, and class 3 included 11 verbal advertisements and 12 pictorial advertisements. The data distribution is shown in Fig. 6. As a result of $k$-means clustering, the data were divided into three color groups, namely, medium color (class 1), light color (class 2), and dark color (class 3).

We also classified the oxy-Hb data according to the classification of the advertisements. The sample sizes of class 1 , class 2 , and class 3 were 120,150, and 138, respectively. The $t$-test was conducted for class 1 versus class 2 , class 1 versus class 3 , and class 2 versus class 3 . The results of the $t$-tests are shown in Figs. 7 and 8. Significant differences were observed between class 1 and class 2 (channel 15) and between class 2 and class 3 (channels 5-8, 10, 11, and 13). No significant differences were observed between class 1 and class 3 .

We further examined the effect of the type of advertisement, i.e., verbal or pictorial, on brain activity. In the verbal case, the sample sizes of the class 1 , class 2 , and class 3 were 54,84 , and 66 , respectively. In the pictorial case, the sample sizes of class 1, class 2, and class 3 were 66 , 

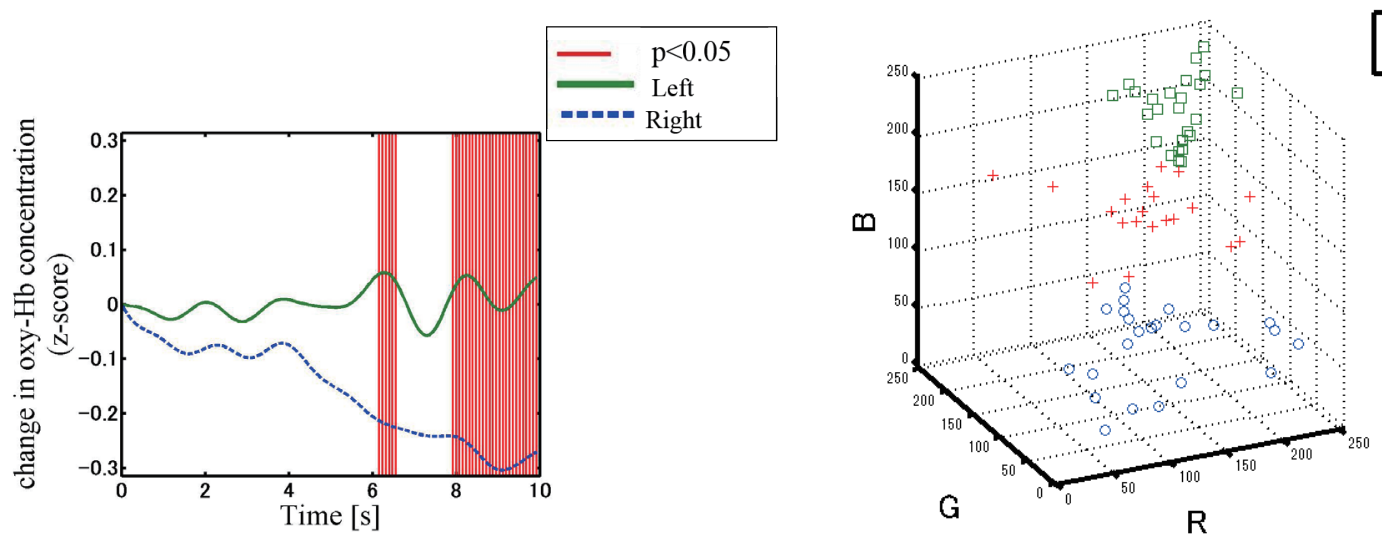

Fig. 5. (Color online) Example of differences in brain activity depending on placement in pictorial advertisement (ch 2).

Fig. 6. (Color online) Distribution of classified data.

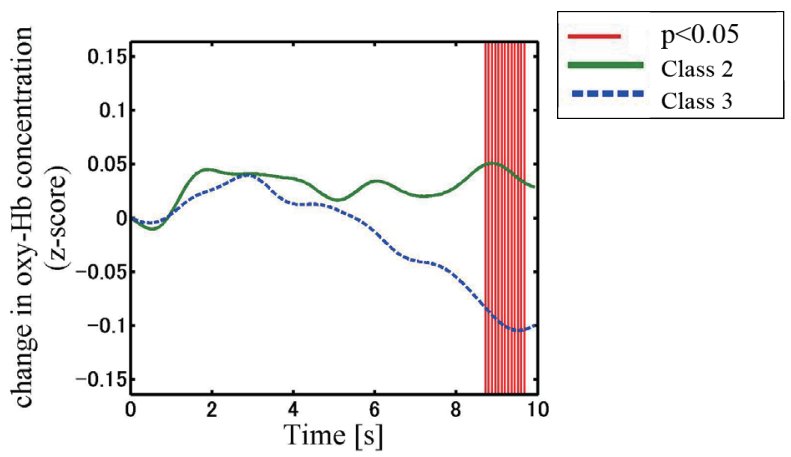

Fig. 7. (Color online) Example of differences in brain activity depending on color in class 1 and class 2 (ch 15).

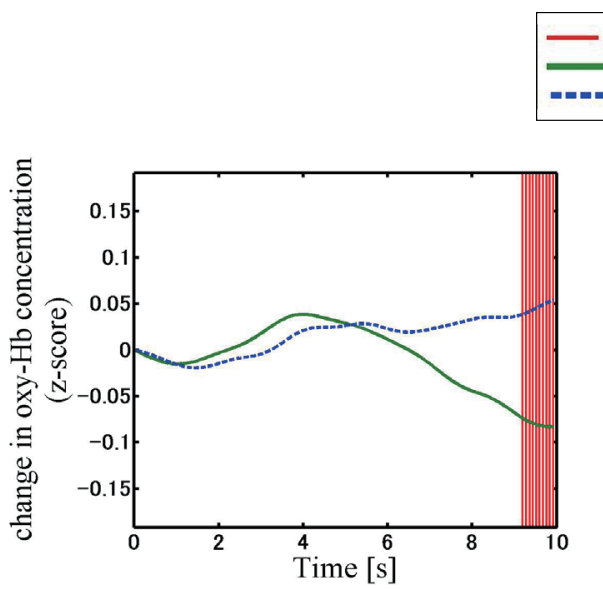

(a)

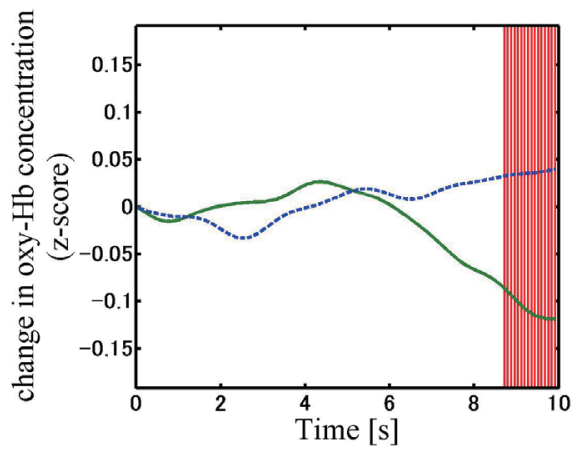

(b)

Fig. 8. (Color online) Example of differences in brain activity depending on color in class 2 and class 3. (a) ch 5 and (b) ch 11 .

66, and 72, respectively. The $t$-test was conducted for the class 1 versus class 2 , class 1 versus class 3 , and class 2 versus class 3 in the pictorial and verbal advertisements. The results of the $t$-tests are shown in Figs. 9-11. 


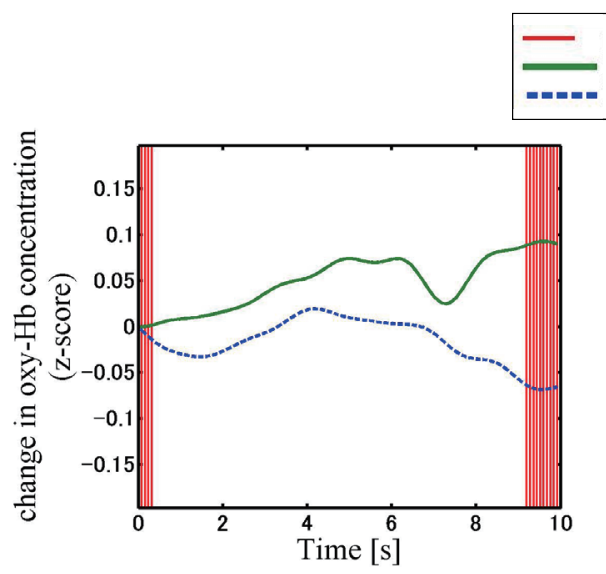

(a)

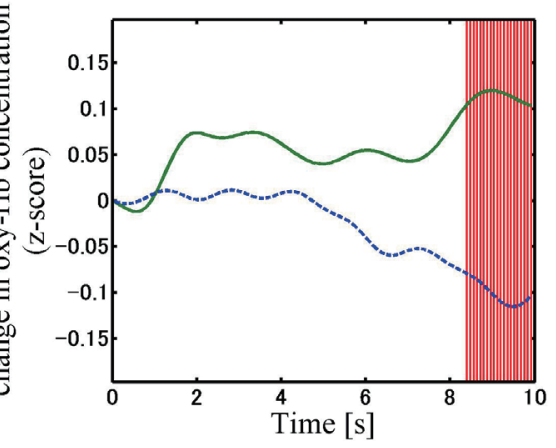

(b)

Fig. 9. (Color online) Example of differences in brain activity depending on color in pictorial advertisement. (a) ch 5 and (b) ch 15 .

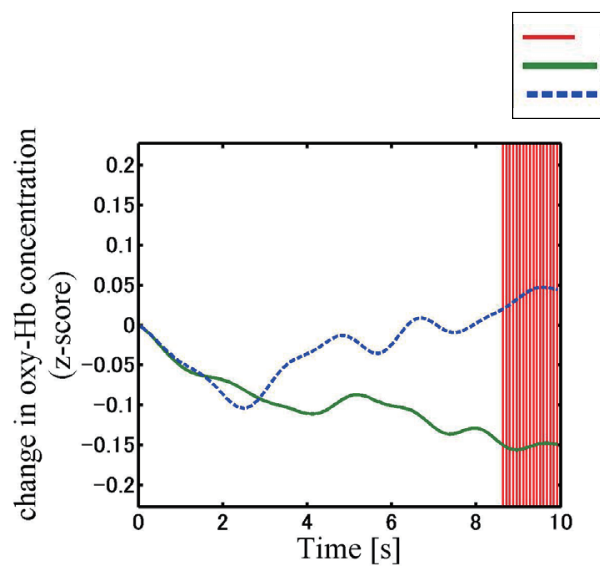

(a)

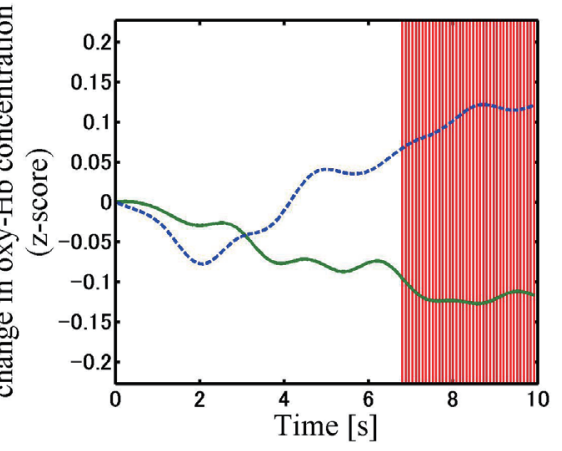

(b)

Fig. 10. (Color online) Example of differences in brain activity depending on color in verbal advertisement in class 1 and class 3. (a) ch 2 and (b) ch 8 .

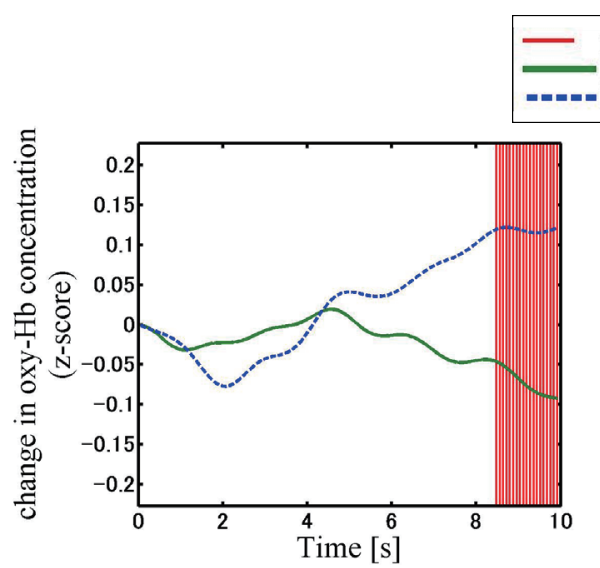

(a)

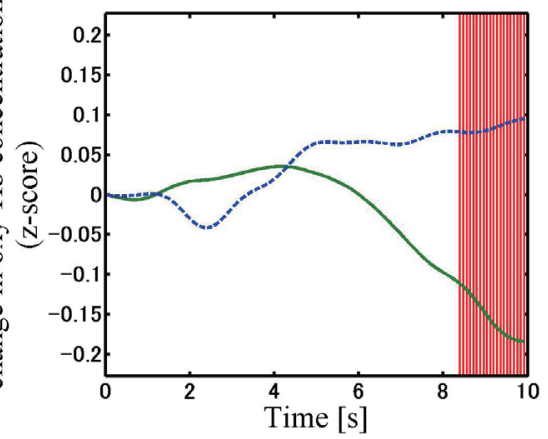

(b)

Fig. 11. (Color online) Example of differences in brain activity depending on color in verbal advertisement in class 2 and class 3. (a) ch 8 and (b) ch 11 . 
In the pictorial advertisements (Fig. 9), significant differences were observed between class 1 and class 2 (channels 4, 5, 14, and 15), while no significant differences were observed between class 1 and class 3, or class 2 and class 3. In the verbal advertisements (Figs. 10 and 11), significant differences were observed between class 1 and class 3 (channels 2, 6, 8, and 11) and between class 2 and class 3 (channels 8 and 11). However, no significant differences were observed between class 1 and class 2 .

\section{Discussion}

As shown in Fig. 4, the oxy-Hb concentration was higher in the group of participants with high ratings, while it was lower in the group with low ratings. Therefore, we assume that brain activity can measure the attractiveness of online advertisements.

As shown in Fig. 5, the oxy-Hb concentration was higher in the case of advertisements placed on the left side of the webpage than those placed on the right side. Therefore, it is clear that pictorial advertisements receive high ratings when placed on the left side of webpages. This is consistent with previous studies ${ }^{(1,13)}$ and is probably due to the fact that visual stimuli perceived in the left visual field is processed by the right brain, which is known to deal with pictorial information.

In our results, no significant differences were observed in the verbal advertisements, which is likely because of the challenges met when detecting differences in brain activity. Furthermore, the results of the questionnaire were biased by low ratings compared with pictorial advertisements.

For the results obtained concerning the color of the advertisement, the concentration of oxy-Hb was higher for advertisements presented with darker colors than for advertisements presented with light colors. Furthermore, these differences were more significant in the verbal advertisements than in the pictorial advertisements. Therefore, we suggest that the verbal advertisements are more affected by color. This may be due to differences in the coloration in our examples. Indeed, the verbal advertisements of class 2 were presented using a dark-colored text on a light-colored background, whereas advertisements of class 3 had the opposite color scheme. A previous study revealed that one can read bright characters on dark backgrounds faster than dark characters on bright backgrounds, ${ }^{(14)}$ which may have influenced these results.

Regarding the pictorial advertisements, many of those in class 2 consisted of a product image on a light-colored background. On the other hand, in many of the class 1 advertisements, products not only stand out, but the product and the background are in harmony. Therefore, for pictorial advertisements, it is preferable that the colors of the product and the background harmonize naturally.

On the basis of the above results, we suggest that people's perceptions of pictorial advertisements are mainly affected by arrangement, while those of verbal advertisements are affected by color. Our findings suggest that the recommended design of pictorial advertisements is to place them on the left side of the webpage and to use natural colors. In the case of verbal advertisements, the best designs should involve bright text on dark backgrounds. However, to determine the optimal design more accurately, various conditions such as gender differences and product categories must be examined in the future. 


\section{Conclusions}

The purpose of this study was to examine the effects of the advertisement's rating, placement, and color on brain activity by using pictorial and verbal advertisements, as a starting point of evaluation and to propose optimal advertisement designs based on the brain activity results.

In our results, oxy-Hb concentration increased when the participants viewed highly rated advertisements, compared with poorly rated advertisements. In addition, significant differences in brain activity were observed depending on the placement and color of the pictorial advertisements and the color of the verbal advertisements. On the basis of these results, we propose that pictorial advertisements are mainly affected by arrangement, whereas verbal advertisements are more affected by color. Thus, the recommended design of pictorial advertisements is to place them on the left side of the webpage and to use natural colors. The optimal design for verbal advertisements is bright text on dark backgrounds.

\section{References}

G. Ryu, E. A. C. Lim, L. T. L. Tan, and Y. J. Han: Electron. Commer. Res. Appl. 6 (2007) 6.

K. Sokolik, R. G. Magee, and J. D. Ivory: J. Interact. Advert. 14 (2014) 31.

3 A. Shaouf, K. Lü, and X. Li: Comput. Human. Behav. 60 (2016) 622.

4 X. Dr’eze and F. Hussherr: J. Interact. Mark. 17 (2003) 8.

5 D. Courbet, M. Fourquet-Courbet, R. Kazan, and J. Intartaglia: J. Compt-media. Comm. 19 (2014) 274.

6 R. Ohme, D. Reykowska, D. Wiener, and A. Choromanska: J. Econ. Psychol. 31 (2010) 785.

7 A. Tversky, D. Kahneman, and R. Choice: Science. 211 (1981) 453.

8 T. D. Wilson, S. Lindsey, and T. Y. Schooler: Psychol. Rev. 107 (2000) 101.

9 G. Jasdzewski, G. Strangman, J. Wagner, K. K. Kwong, R. A. Poldrack, and D. A. Boas: Neuroimage 20 (2003) 479.

10 T. Yamada, S. Umeyama, and K. Matsuda: PLoS One 7 (2012) e50271.

11 Y. Tong and B. deB. Frederick: Neuroimage 53 (2010) 553.

12 M. Balconi, E. Grippa, and M. E. Vanutelli: Brain Cognit. 95 (2015) 67.

13 C. Janiszewski: J. Consum. Res. 15 (1988) 199.

14 M. V. Mclean: Hum. Factors 7 (1965) 521.

\section{About the Authors}

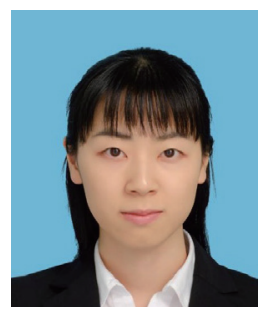

Chikaho Kurahashi received her B.E. degree in Intellectual Information Engineering from the University of Toyama, Toyama, Japan, in 2016. Currently, she is a graduate student at the Department of Intellectual Information Engineering, Graduate School of Science and Engineering for Education, University of Toyama. 


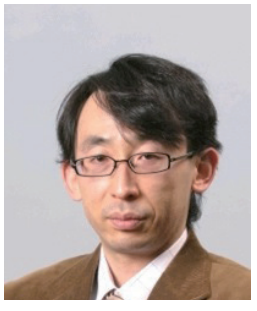

Tadanobu Misawa received his B.E., M.E., and Ph.D. degrees from Kanazawa University, in 1999, 2001, and 2004, respectively. He was a doctoral fellow with the Kanazawa Institute of Technology in 2004. From 2005 to 2008, he was an assistant professor with the School of Management, Tokyo University of Science. He is currently an associate professor with the Graduate School of Science and Engineering, University of Toyama. His research interests are artificial intelligence and brain-computer interface.

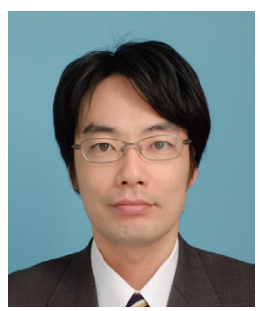

Kazuya Yamashita received his B.E. and M.E. degrees in Intellectual Information Engineering from University of Toyama in 2004 and 2006, respectively. Since 2006, he has been with University of Toyama where he is presently an assistant professor in the Faculty of Engineering. His research interests include formal language theory, automaton theory and algorithms. 